\title{
Metabolic alterations in meningioma reflect the clinical course
}

\author{
Waseem Masalha ${ }^{1,2^{*}}$ (D), Karam Daka ${ }^{1,2}$, Jakob Woerner ${ }^{3}$, Nils Pompe ${ }^{3}$, Stefan Weber ${ }^{3}$, Daniel Delev ${ }^{4}$, \\ Marie T. Krüger ${ }^{5}$, Oliver Schnell ${ }^{1,2}$, Jürgen Beck ${ }^{1,2}$, Dieter Henrik Heiland ${ }^{1,2+}$ and Juergen Grauvogel ${ }^{1,2+}$
}

\begin{abstract}
Background: Meningiomas are common brain tumours that are usually defined by benign clinical course. However, some meningiomas undergo a malignant transformation and recur within a short time period regardless of their World Health Organization (WHO) grade. The current study aimed to identify potential markers that can discriminate between benign and malignant meningioma courses.

Methods: We profiled the metabolites from 43 patients with low- and high-grade meningiomas. Tumour specimens were analyzed by nuclear magnetic resonance analysis; 270 metabolites were identified and clustered with the AutoPipe algorithm.

Results: We observed two distinct clusters marked by alterations in glycine/serine and choline/tryptophan metabolism. Glycine/serine cluster showed significantly lower WHO grades and proliferation rates. Also progressionfree survival was significantly longer in the glycine/serine cluster.

Conclusion: Our findings suggest that alterations in glycine/serine metabolism are associated with lower proliferation and more recurrent tumours. Altered choline/tryptophan metabolism was associated with increases proliferation, and recurrence. Our results suggest that tumour malignancy can be reflected by metabolic alterations, which may support histological classifications to predict the clinical outcome of patients with meningiomas.
\end{abstract}

Keywords: Tumour metabolism, Meningioma

\section{Background}

Meningiomas are common brain tumours in adults; they account for $13-26 \%$ of all intracranial tumours [1]. The 2016 World Health Organization (WHO) classification divided meningiomas into three histological grades (I to III) and described 16 histopathological subtypes [2]. These WHO grades have presented a significant correlation with clinical outcomes in several recent studies. The majority of meningiomas are benign tumours (90\%)

\footnotetext{
* Correspondence: waseem.masalha@uniklinik-freiburg.de

${ }^{\dagger}$ Dieter Henrik Heiland and Juergen Grauvogel contributed equally to this work.

'Department of Neurosurgery, University Medical Center Freiburg, Breisacher Straße 64, 79106 Freiburg, Germany

${ }^{2}$ Faculty of Medicine, University of Freiburg, Freiburg im Breisgau, Germany Full list of author information is available at the end of the article
}

that are mainly treated by surgery, followed by an innocent clinical course [1]. Atypical meningiomas (WHO grade II) reveal a worse clinical outcome due to higher recurrence rates of up to $30-40 \%$ [2]. In addition, a small subset of meningiomas $(1-2.8 \%)$ is classified as anaplastic; they show a particularly aggressive clinical course and a recurrence rate of nearly $100 \%$ [1].

A few benign meningiomas (WHO grade I) can transform into an aggressive growth pattern and recur after a short period of time. To address the variety of clinical outcomes within the WHO grade I group, multiple research groups have investigated the genetic landscape of meningiomas and have shown region-specific genomic alterations [3]. The authors have identified five genetic subgroups based on transcriptional similarity and

(c) The Author(s). 2021, corrected publication 2021. Open Access This article is licensed under a Creative Commons Attribution 4.0 International License, which permits use, sharing, adaptation, distribution and reproduction in any medium or format, as long as you give appropriate credit to the original author(s) and the source, provide a link to the Creative Commons licence, and indicate if changes were made. The images or other third party material in this article are included in the article's Creative Commons licence, unless indicated otherwise in a credit line to the material. If material is not included in the article's Creative Commons licence and your intended use is not permitted by statutory regulation or exceeds the permitted use, you will need to obtain permission directly from the copyright holder. To view a copy of this licence, visit http://creativecommons.org/ licenses/by/4.0/. The Creative Commons Public Domain Dedication waiver (http://creativecommons.org/publicdomain/zero/1. 0/) applies to the data made available in this article, unless otherwise stated in a credit line to the data. 
associated mutational patterns. Meningiomas most frequently contained an NF2, SMARCB1 (Group1), TRAF7/ KLF4 (Group2), and PI3K (Group3) mutation, which drive WNT pathway activation. In another group, there was Hedgehog pathway activation detected by transcriptional profiles and mainly based on mutations in genes such as SMO [4]. The last subgroup comprised meningiomas that showed a distinct mutation in the POLR2A gene followed by dysregulated RNA synthesis [3, 5]. others suggested that malignant meningiomas have different DNA methylation patterns than other types of meningiomas and that the differently methylated genes could serve as diagnostic biomarkers for malignant transformation [6]. The importance of TERT promoter mutations and their potential use as biomarkers to identify meningiomas at risk of malignant transformation was also reported [7].

Besides genomic alterations, tumour metabolism has recently been described as a hallmark driver of malignancy and tumour development. Different tumour entities, including brain and other solid tumours, have shown numerous metabolic alterations. A strong association between altered tumourmetabolism and chromosomal instability has been reported [8]. These metabolic alterations were detected independently of the WHO grade $[9,10]$. Serna et al. [11] showed that metabolic aggressiveness is driven by alterations in the expression of IGF1R (insulin-like growth factor 1 receptor), which is involved in the regulation of glycolysis. Following the hypothesis of altered glycolysis in meningiomas, researchers have shown that different components of glycolysis are transformed, including phosphofructokinase (PFK) and lactate dehydrogenase (LDH), both of which were significantly increased in anaplastic meningioma compared with other histological subtypes. In addition, there were alterations in tryptophan metabolism, a phenomenon that forced the immune-escape mechanism to increase kynurenine pathway activity $[12,13]$. Today, increased opportunities in bioinformatics and metabolic profiling have allowed detecting associations between metabolic networks and clinical parameters to predict metabolic patterns and associated clinical outcomes. Our aim was to identify benign and potentially malignantly transformed meningiomas within the defined WHO grade I by metabolic profiling and computational analysis.

\section{Methods}

\section{Contact for reagent and resource sharing}

Further information and requests for resources, raw data, and reagents should be directed to and will be fulfilled by DH Heiland (dieter.henrik.heiland@uniklinikfreiburg.de). A full table of all materials is given in the supplementary information.

\section{Ethical approval}

For this study, we included 43 patients who underwent surgery at the Department of Neurosurgery of the University Medical Center Freiburg. The local ethics committee of the University of Freiburg approved data evaluation, imaging procedures, and the experimental design (protocols 100,020/09 and 5565/15). The methods were carried out in accordance with the approved guidelines. Written informed consent was obtained from each patient. The studies were approved by an institutional review board.

\section{Imaging, tissue collection, and histology}

Tumour tissue was sampled from the meningioma core, snap-frozen in liquid nitrogen immediately after resection, and processed for further metabolic analysis. Representative tissue from all samples were fixed using $4 \%$ phosphate-buffered formaldehyde and embedded in paraffin following standard procedures. Hemotoxylin and eosin (H\&E) staining was performed on $4 \mu \mathrm{m}$ paraffin sections using standard protocols. This staining confirmed the correct sampling.

\section{Metabolite extraction and hydrogen nuclear magnetic resonance ( $\left.{ }^{1} \mathrm{H}-\mathrm{NMR}\right)$ analysis}

Metabolites were extracted with $400 \mu \mathrm{L}$ ice-cold $80 \%$ methanol and $400 \mu \mathrm{L}$ ice-cold water, homogenized with a tissue grinder (VWR, Radnor, PA, USA), sonicated at $1{ }^{\circ} \mathrm{C}$, then centrifuged at $15,000 \mathrm{~g}$ for $20 \mathrm{~min}$ to remove protein. Extracts were dried by lyophilization and resuspended in $650 \mu \mathrm{L}$ deuterated water as described by Beckonert et al [14] Six-hundred microliters of the suspension was transferred to NMR tubes for the subsequent NMR procedure. ${ }^{1} \mathrm{H}-\mathrm{NMR}$ spectra were collected at the Institute of Physical Chemistry of the University of Freiburg with a Bruker Avance III HDX $600-\mathrm{MHz}$ NMR spectrometer (Bruker, Rheinstetten, Germany), equipped with a PABBO BB/19F-1H/D ZGRD probe head. Each individual spectrum was recorded with two dummy scans and 32 scans with $64 \mathrm{k}$ points in the time domain. The sweep width was set to $16.02 \mathrm{ppm}$ with an offset of $4.691 \mathrm{ppm}$. This resulted in an acquisition time of $3.4 \mathrm{~s}$ for each scan and a dwell time of 52 microseconds. The relaxation delay was set to $2 \mathrm{~s}$ for acquisition, and the water signal was suppressed by an excitation sculpting scheme [15].

\section{Postprocessing of metabolic data}

To adjust the spectra from multiple batches, spectra were manually aligned by setting the peak of L-lactic acid at $1.310 \mathrm{ppm}$. All acquisition and processing of the spectra were performed with TopSpin 3.2 patch level 6. A detailed description of the methods was given in a recently published study by Heiland et al [16] All spectra 
were analyzed with the software package "batman," an R-software-based tool for metabolite detection in complex spectra [17]. This tool fits a predefined list of metabolites by a Bayesian approach. Hao et al. [17] provided a detailed description of the batman algorithm. Normalization of the spectra was performed by the pseudo-counted quantile ( $\mathrm{pQ}$ ) normalization algorithm integrated in the KODAMA package. Further processing of metabolic data is described in the subsequent subsections.

\section{Cluster analysis}

Normalized metabolic data were processed with AutoPipe (https://github.com-/heilandd/AutoPipe), a software package for automated unsupervised clustering. First, the number of subgroups was computed by the Partitioning Around Medoids (PAM) algorithm (Cluster number $k=2-12$ ). To identify the optimal number of clusters, we calculated the mean silhouette width of each cluster composition. Next, to identify the core samples of each cluster, we removed samples with a negative silhouette width from further analysis. We then used either the PAMR algorithm [18], a machine-learning-based method, or a generalized linear model [19] to identify characteristic up- or downregulated metabolites of each subgroup.

\section{Weighted correlation network analysis (WCNA)}

WCNA is a robust tool for integrative network analysis and has been used in recent studies [20-22]. It is based on a scaled-topology-free-based network approach and uses the topological overlapping measurement to identify corresponding modules. These modules were analyzed by their eigengene correlation to each metabolite. The correlation of the intramodular connectivity (kME) and metabolites was used as input for a "Cluster of Clusters Analysis." This analysis integrates expression modules and metabolites, which presents equal correlation values (kME and metabolite intensity values). A detailed description of WCNA is given in a previous publication [23].

\section{Functional analysis}

Metabolic data was processed by pathviewer, an $\mathrm{R}$ package that includes Kyoto Encyclopedia of Genes and Genomes (KEGG) pathway maps [24]. Expression data (as described above) and normalized, log2transformed, and median-centered metabolic data were integrated in the pathviewer algorithm. Enrichment analysis of metabolic data was performed with the DOSE package and the web-based tool MetaboAnalyst 3.0 (www.metaboanalyst.ca).

\section{Survival analysis}

Progression-free survival analysis was conducted using Kaplan-Meier analysis, with the betweed-cluster differences analyzed using a log-rank test.

\section{Results \\ Meningiomas revealed two distinct metabolic clusters}

We started our investigation by purifying metabolites from 43 meningiomas localized at different anatomical regions, including the convexity in 12 cases $(27.9 \%)$, the falcine site in 6 cases (13.9\%), the sphenoid ridge in 6 cases $(13.9 \%)$, the frontobasal region in 9 cases $(20.9 \%)$, the petroclival region in 2 cases $(4.6 \%)$, the spinal site in 3 cases $(6.9 \%)$, and 5 cases at other regions. The histopathological analysis showed 28 patients with WHO grade I meningiomas (65.1\%), 12 with WHO grade II meningiomas $(27.9 \%)$, and 3 patients with WHO grade III meningiomas (6.9\%). Two patients with a WHO grade II meningioma and 3 patients with a WHO grade III meningioma showed a tumour recurrence within 1 year. Twenty-four (55.9\%) patients had a proliferation index (MIB-1) below 5\% and 19 (44.1\%) patients had a proliferation index (MIB-1) above 5\%. A gross total resection (Simpson grade $1+2$ ) was achieved in 37 patients and a subtotal resection (Simpson grade $3+4$ ) was achieved in 6 patients. A detailed overview of all parameters can be found in Table 1.

Metabolites were analyzed using NMR and processed by a comprehensive computational analysis (Fig. 1). Representative MRI and histological staining's are illustrated in Fig. 1b-c. We first conducted an unsupervised cluster analysis of the top 100 most variable metabolites by PAM clustering. The optimal number of clusters was defined by maximal mean silhouette widths (Fig. 2a, b). The analysis revealed two distinct clusters. In the first cluster, named Metabolic Cluster I, we found upregulated glycine/serine metabolism with the major signature metabolites glycine, serine, and arginine. Patients in this cluster were exclusively histological grade I and showed a significantly lower rate of edema $(p<0.05)$ and a low proliferation rate (mean 1.2, interquartile range [IQR] $0.3, p<0.05)$ compared to cluster II. The second cluster (Metabolic Cluster II) was easily separable into two subclusters by PAM clustering. One subcluster contained patients with a medium proliferation rate of 2.1 (IQR $0.7)$ and increased edema compared with Cluster I $(p<$ 0.05; Fig. 2a, b). The pathway that separated the clusters was choline metabolism. For the second subcluster, we found increased tryptophan metabolism and also a strong activation of the choline pathway. Furthermore, the proliferation rate was massively increased (mean 11.7, IQR 4.3, $p<0.05$ ), and there was edema in most of the patients compared to patients in cluster I. We found a high incidence of meningioma WHO grade II $(n=12)$ 
Table 1 Patients data

\begin{tabular}{|c|c|c|c|}
\hline Parameter & $\begin{array}{l}\text { Cluster } 1 \\
N=15\end{array}$ & $\begin{array}{l}\text { Cluster } 2 \\
N=28\end{array}$ & Significance level (p) \\
\hline \multicolumn{4}{|l|}{$\operatorname{Sex}(N, \%)$} \\
\hline Male $(N=10)$ & $2(14.2 \%)$ & $8(28.5 \%)$ & $0.45^{* * * *}$ \\
\hline Female $(N=33)$ & $13(86.6 \%)$ & $20(71.4 \%)$ & \\
\hline Age (mean, SD) & $62.3 \pm 13.1$ & $64.4 \pm 12.8$ & $0.62^{*}$ \\
\hline Size $\left(\mathrm{cm}^{3}\right)$ (mean, SD) & $48.3 \pm 51.8$ & $88.6 \pm 103.3$ & $0.09^{*}$ \\
\hline \multicolumn{4}{|l|}{ Peritumoral edema (N, \%) } \\
\hline high & $4(26.6 \%)$ & $18(64.3 \%)$ & $0.02^{* * *}$ \\
\hline low & $11(73.4 \%)$ & 10(35.7\%) & \\
\hline \multicolumn{4}{|l|}{ Location (N, \%) } \\
\hline falx & $3(20 \%)$ & $3(10.7 \%)$ & $0.64^{* * * *}$ \\
\hline convexity & $2(13.3 \%)$ & 10(35.7\%) & $0.16^{* * * *}$ \\
\hline frontobasal & $4(26.6 \%)$ & $5(17.8 \%)$ & $0.69^{* * * *}$ \\
\hline sphenoid wing & $2(13.3 \%)$ & $4(14.2 \%)$ & $1^{* * *}$ \\
\hline petroclival & $1(6.6 \%)$ & $1(3.5 \%)$ & $1^{* * * *}$ \\
\hline spinal & $2(13.3 \%)$ & $1(3.5 \%)$ & $0.11^{* * * *}$ \\
\hline others & $1(6.6 \%)$ & $4(14.2 \%)$ & $0.64^{* * * *}$ \\
\hline \multicolumn{4}{|l|}{ Simpson grade (N, \%) } \\
\hline Grade 1 & $5(33.3 \%)$ & $7(25 \%)$ & $0.74^{* * * *}$ \\
\hline Grade 2 & $9(60 \%)$ & $16(57.1 \%)$ & $1^{* * *}$ \\
\hline Grade 3 & $0(0 \%)$ & $3(10.7 \%)$ & $0.54^{* * * *}$ \\
\hline Grade 4 & $1(6.6 \%)$ & $2(7.1 \%)$ & $1^{* * * *}$ \\
\hline Preoperative KPS (median, 95\% Cl) & $80 \% \mathrm{Cl}(70-80 \%)$ & $70 \%$ Cl(\%60-80\%) & $0.01^{* *}$ \\
\hline Postoperative KPS (median, 95\% CI) & $90 \% \mathrm{Cl}(80-90 \%)$ & $70 \%$ Cl(50-90\%) & $0.007^{* *}$ \\
\hline \multicolumn{4}{|l|}{ Proliferation index (N, \%) } \\
\hline $\mathrm{MlB} 1<5 \%$ & 15(100\%) & $9(32.1 \%)$ & $0.01 * * * *$ \\
\hline MIB1 > 5\% & $0(0 \%)$ & 19(67.9\%) & \\
\hline \multicolumn{4}{|l|}{ Pathology (N, \%) } \\
\hline WHO grade I & $15(100 \%)$ & $13(46.4 \%)$ & $0.001 * * * *$ \\
\hline WHO grade $\|+I I I$ & $0(0 \%)$ & 15(53.6\%) & \\
\hline
\end{tabular}

$S D$ standard deviation

$\mathrm{Cl}$ confidence interval

KPS Karnofsky score

* T-test

**Wilcoxon test

***Chi-squared test

****Fisher's exact test

and III $(n=3)$ but also meningioma WHO grade I $(n=$ 13) (Fig. 2a, b). Kaplan-Meier analysis showed a significant longer progression-free survival in favour of cluster I $(p=0.0083)$, suggesting that metabolic alterations may reflect the clinical course of the disease, Fig. 3.

\section{WCNA of metabolic profiles in Meningiomas}

We aimed to characterize the metabolic pathways in detail and performed a WCNA of metabolic profiles. There were five metabolic modules (Fig. 2c). Three modules showed a strong association with the WHO grade based on a logistic model that included WHO grade and module eigengene. From that model, we considered module GI (associated with WHO grade I, $R=0.25, p_{\text {adj }}=0.05$ ), module GII (associated with WHO grade II, $R=0.26$, $p_{\text {adj }}=0.05$ ), and module GIII (associated with WHO grade III, $R=0.28, p_{\text {adj }}=0.01$ ) for further analysis. The other modules failed to achieve a significant correlation with the WHO grade. First, we analyzed the correlation between the GI module eigengene and clinical features (Figure S1A). We found a significant negative correlation between module GI and perifocal edema $(R=-0.3$, 


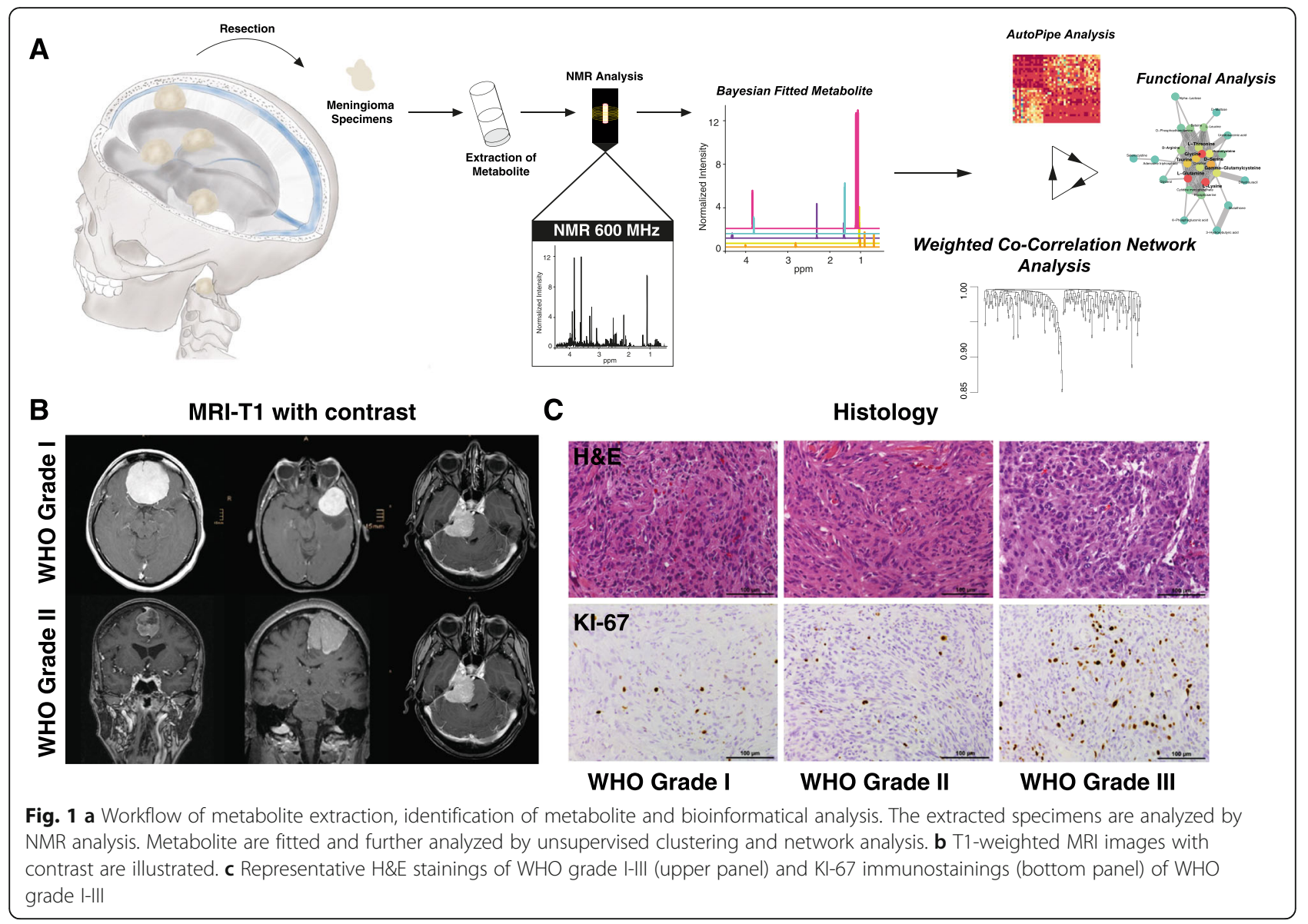

$\left.p_{\text {adj }}=0.01\right)$ and the histologically defined atypical meningiomas $\left(R=-0.38, p_{\text {adj }}=0.03\right)$, which confirmed our NMR findings. Meningiomas with a module GI signature were mostly observed in the convexity $\left(R=-0.32, p_{a d j}=\right.$ $0.04)$. The histological subtype of a "transitional meningioma" was highly significantly associated with the GI signature $\left(R=-0.41, p_{a d j}=0.001\right)$. Next, we examined the enrichment of metabolic pathways in each module. A metabolite set enrichment analysis (MSEA) showed a significant upregulation of the glycine/serine pathway (Enrichment Score $=1.75, p=0.0067$ ) and glutamate metabolism (Enrichment Score $=1.52, p=0.047$ ) (Figure S1B). We computed a network based on the metabolites of module GI. Connections within the network were based on intramodular connectivity. From that network, we identified the amino acids serine and glycine and the metabolite creatine as key metabolic players in grade I meningiomas.

In the next step, we examined the correlation between the GII module eigengene and clinical features (Figure S2A). There was a significant correlation between module GII and perifocal edema $\left(R=0.25, p_{a d j}=0.05\right)$ and the histologically defined atypical meningioma $\left(R=0.41, p_{a d j}=\right.$ $0.02)$. There was no correlation between localization and the module eigengene. The metabolic network of module GII identified tryptophan and kynurenic acid as hub metabolites (Figure S2B). In line with the network findings, a metabolic pathway analysis uncovered upregulation of the tryptophan metabolism pathway (Enrichment Score = 1.83, $p=0.0093$; Fig. 2c). To characterize module GIII, we examined the correlation between module eigengene and clinical features (Fig. S2D). No clinical feature was associated with this module, except the WHO grade $(R=0.28$, $\left.p_{\text {adj }}=0.01\right)$. In addition, there was no correlation between localization and module eigengene. We next computed a network of metabolites for module GIII. Choline, isoleucine, and sphingosine were hub metabolites, (Figure S2E). In line with the network findings, a metabolic pathway analysis uncovered an upregulation of the choline metabolism pathway (Enrichment Score $=1.06, p=0.0014$ ).

\section{Discussion}

Due to a favorable clinical course, most meningiomas are classified as benign. Nevertheless, $20 \%$ of patients within this subgroup present a tumour recurrence, even after gross total resection $[1,25]$. These tumour subtypes are difficult to identify and challenging for future treatment. To better predict their clinical outcome, researchers 
A

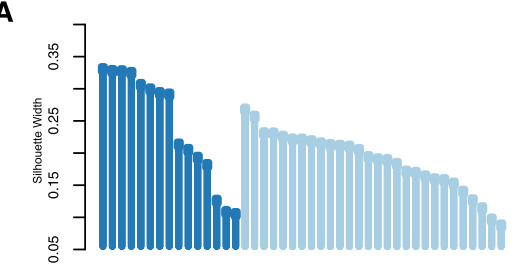

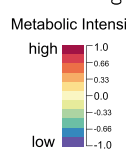

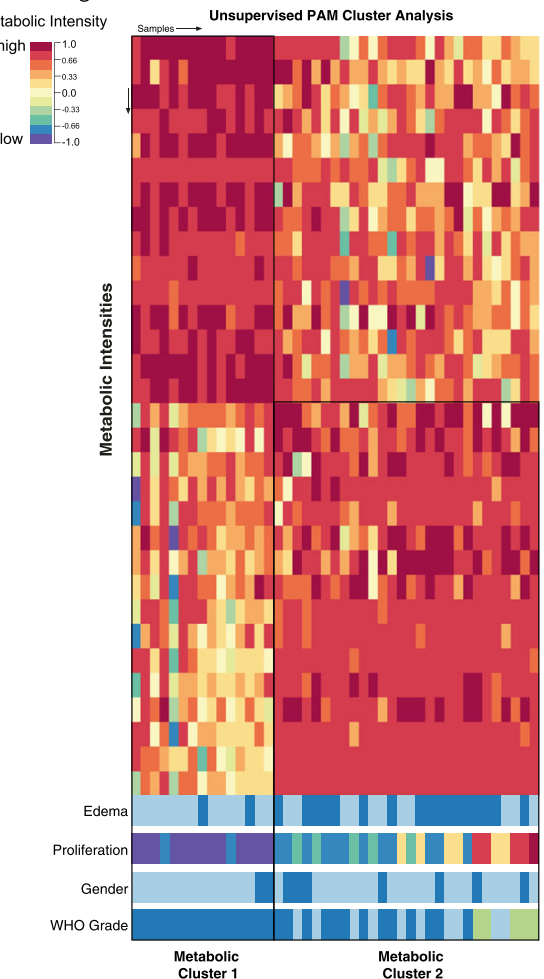

B
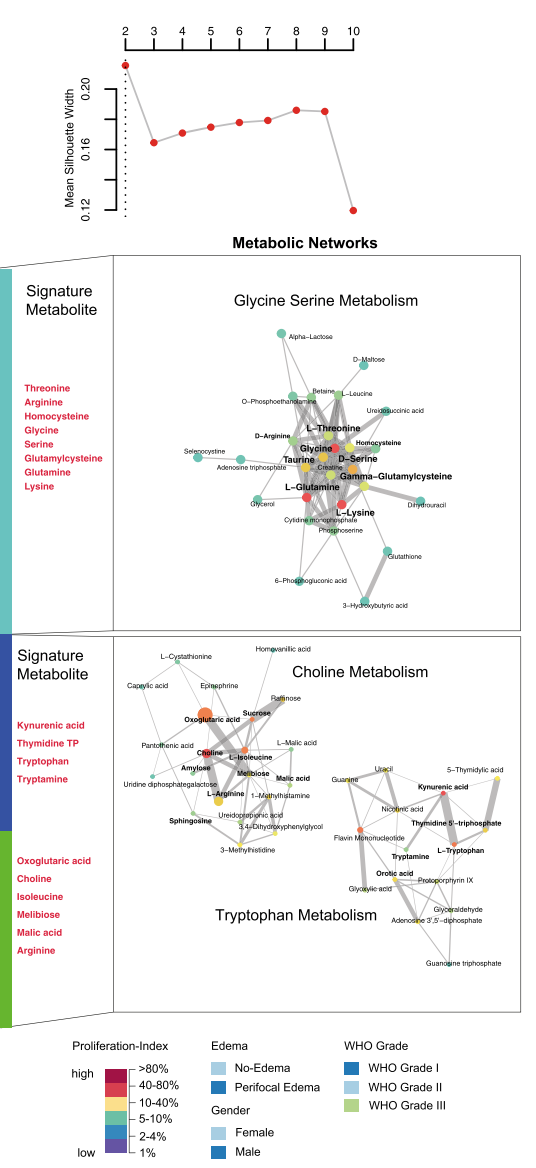

C

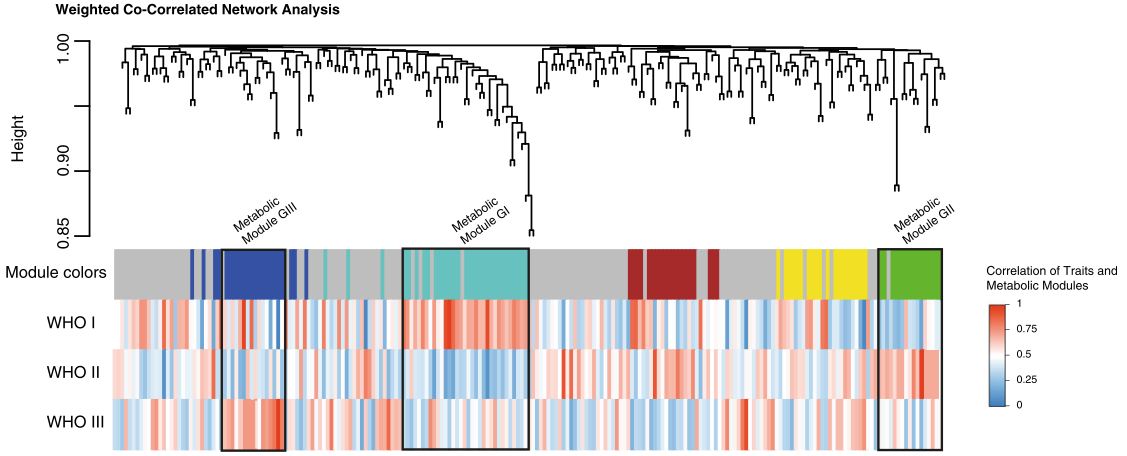

Fig. 2 a In the upper plot, the silhouette widths of both clusters are shown ordered by different cluster subgroups. In the middle panel, a heatmap illustrate the metabolic intensity of the top ranked metabolites. At the bottom panel, the information on clinical properties is available. These are color-coded and explained in the side panel. $\mathbf{b}$ In the upper panel, the plot illustrates the optimal number of clusters. The optimal number of clusters was achieved by PAM clustering from 2 to 10 number of clusters by calculating the mean silhouette widths. In the bottom panel, a network analysis of the metabolic pathways highly enriched in cluster 1 and 2 respectively is given. c A weighted correlation network analysis (WGNC) was performed to explore the associated clinical features and molecular/metabolic, further detailed interpretation of all identified modules is given in the supplementary data

have performed metabolic analysis by high resolution magic angle spinning (HR-MAS) and reported a correlation between metabolic and WHO graduation of meningiomas [9]; however, these findings were limited by a small number of observed metabolites. We aimed to investigate a global metabolic profiling to identify specific 


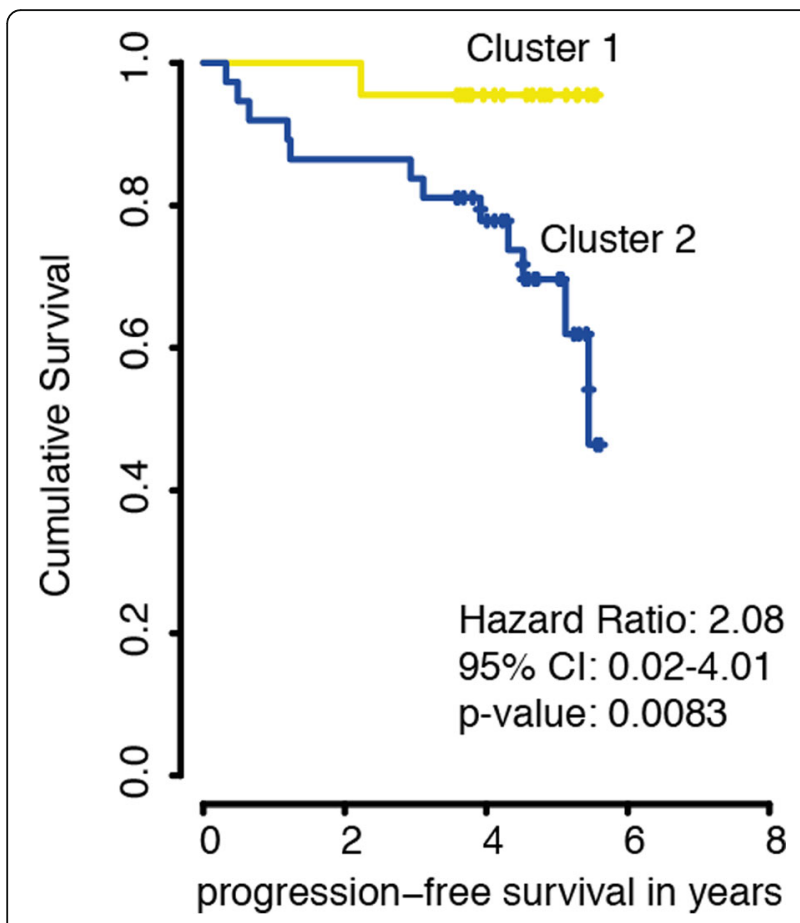

Fig. 3 Kaplan-Meier Kurve for Prgression-free survival based on different Clusters (Cluster 1 in yellow vs Cluster 2 in blue)

subgroups that are driven by unique metabolic alterations and associated with WHO grades. We performed ex vivo metabolic profiling by NMR spectroscopy of meningioma specimens, including all WHO grades (I, II, and III), followed by computational detection of metabolites and cluster analysis.

Our cluster analysis identified two major metabolic clusters, of which one cluster was divided into two subclusters of meningiomas based on the top 100 most variable metabolites. The first metabolic cluster was highlighted by serine and glycine metabolism, which provides a basis for synthesis of other amino acids, proteins, and lipids. Their metabolism could increase the antioxidative capacity and reduce cellular reactive oxygen species (ROS). In particular, the glycine cleavage system fuels mono-carbon metabolism within a complex cellular network based on folate compound reactions, which play a central role in cellular redox balance over modulation of the $\mathrm{NADP}^{+} / \mathrm{NADPH}-$ ratio [26]. This network is also an essential component of methylation reactions that may be relevant for the maintenance of the cellular epigenetic status [27]. In our study, serine and glycine metabolism was associated with benign meningiomas (WHO grade I) and a significantly low proliferation index $(p=0.01)$. This cluster was also accompanied by significantly lower edema based on magnetic resonance imaging ( $p=0.02$; Fig. 2 , Table 1$)$ compared with higher grades. The role of glycine in meningiomas has been controversially discussed. While some authors have reported a complete absence of glycine in aggressive meningioma [28], others have shown a significantly increased glycine/alanine ratio. Furthermore, a significantly increased ratio of glycine to glutamine/glutamate in rapidly recurrent meningiomas and an increased glycine value in benign meningioma has been observed $[29,30]$. By contrast, Monleon and colleagues found increased glycine levels in atypical meningiomas due to hypothesized increased angiogenesis [9]. These discrepancies with regard to glycine levels suggest the existence of more complex subgroups within the pathological delineation of grade I meningiomas. In line with published data, we detected altered glycine/serine metabolism in a subgroup of benign meningiomas. Other clusters also contain grade I tumours, which results in inaccurate individual metabolic levels within grade I meningiomas.

The second metabolic cluster was defined by choline and tryptophan metabolism. Choline is an important component of the cell membrane. Higher choline concentrations are usually present in brain tumours compared with normal brain tissue. Thereby, choline acts as a brain tumour marker [31]. Elevated choline peaks can also be detected in regions with high tumour cell density, in which the tissue is not necessarily anaplastic [32]. The increased rate of proliferation, which we observed in tumours of the second cluster, consequently drives the choline metabolism due to increased demand of membrane components. Previous findings have suggested that choline levels are significantly increased in benign meningioma with an aggressive clinical course $[9$, 11]. Further, Mishra and colleagues reported high choline levels in malignant meningioma [33]. By contrast, others have reported a prominent choline level in WHO grade I meningioma. While they did not observe differences in choline concentrations within all WHO grades, they only included a small number of WHO grade II + III meningiomas in their study [29]. We further identified altered tryptophan metabolism; this amino acid is mainly metabolized by three pathways: the serotonin (5hydroxytryptamine [5-HT]) pathway, the kynurenine (KYN) pathway, and the tryptamine pathway [34]. Researchers have reported that an overexpression of the tryptophan catabolizing enzymes indoleamine 2,3-dioxygenase $1 / 2$ or tryptophan 2,3-dioxygenase are associated with tumour progression. These two enzymes convert tryptophan into kynurenine $[35,36]$, which is involved in the immune escape mechanism $[37,38]$. This indicates that the tryptophan and the kynurenine pathways play an important role in tumour progression and immune response. Researchers have confirmed the increased tryptophan metabolism in high-grade meningiomas $[12,13]$. In line with the reported findings, we confirmed the increased tryptophan metabolism in high-grade meningiomas (grade 
II and III) but also identified benign meningiomas with upregulated choline and tryptophan metabolism. These tumours were significantly associated with a shorter PFS in our study ( $p=0.0083$ ) (Fig. 3). This insight provides a possible rationale suggesting that some benign classified meningioma manifest a less favorable course than others.

Taken together our findings suggest an important role for tumour metabolism in meningiomas that only partially overlaps with respect to their histological grading. We addressed the questions as to how metabolic changes in meningiomas can provide a possible explanation for different clinical features within these histologically classified tumours. Our metabolic profiling revealed distinct subgroups marked by different activated metabolic programs. Patients with predominant activation of glycine/serine metabolism showed favorable grading and a low proliferation index. The second metabolic subgroups were marked by upregulated choline and tryptophan metabolism; it mainly contained high-grade meningiomas but also some lowergrade tumours. The current study is limited by the relatively low number of enrolled patients and the short follow-up time. However, although WHO grade II and III meningiomas are rare, we were able to include 15 patients in this study. A re-evaluation with a longer follow-up should be carried out to confirm these results.

\section{Conclusion}

In summary, our results suggest that tumour malignancy is reflected by metabolic alterations that can support histological classifications in order to predict the clinical outcome of patients with meningiomas. In our study, we investigated choline as a metabolic marker to predict early recurrence and reduced PFS, which is a welldetectable metabolite in in-vivo MR spectroscopy. Further studies are needed to validate this potential correlation in a larger cohort, which would provide a clinical implication for MR spectroscopy in meningioma.

\section{Supplementary Information}

The online version contains supplementary material available at https://doi. org/10.1186/s12885-021-07887-5.

Additional file 1: Supplementary Figure 1. A) Heatmap of correlation of module eigengene of module $\mathrm{Gl}$ and clinical features. B) Metabolic set enrichment analysis (MSEA) of module GI and KEGG pathways. C)

Network of metabolites of module Gl, nodes and edges were defined by correlation coefficients between all metabolites of module GI. D) A volcano plot reveals differential metabolic intensities between WHO grade I and III meningioma.

Additional file 2: Supplementary Figure 2. A) Heatmap containing correlations of module eigengene (module GII) and clinical features. B) Network of metabolites of module GIl, nodes and edges were defined by correlation coefficients between all metabolites of module Gll. C) Metabolic set enrichment analysis (MSEA) of module Gll and KEGG pathways. D) Correlation of module eigengene (module GIII) and clinical features. E) Network analysis of module GIII, nodes and edges were defined by correlation coefficients between all metabolites. F) Metabolic set enrichment analysis (MSEA) of module GIII and KEGG pathways.

Additional file 3: Supplementary Figure 3. A-B) Scatter plot of different metabolic intensities between WHO grade I and II (A) and II and III $(B)$, respectively. Colors indicate the corrected $p$-values, cyane points with FDR $p<0.05$.

\section{Abbreviations}

NMR: Nuclear magnetic resonance; WHO: World Health Organization; IGF1R: Insulin-like growth factor 1 receptor; PFK: Phosphofructokinase; LDH: Lactate dehydrogenase; H-NMR: Hydrogen Nuclear Magnetic Resonance; ppm: Parts per million; pQ: Pseudo-counted quantile; WCNA: Weighted correlation network analysis; kME: Intramodular connectivity; MSEA: Metabolite set enrichment analysis; HR-MAS: High resolution magic angle spinning; ROS: Reactive oxygen species; 5-HT: 5Hydroxytryptamine; KYN: Kynurenine; PFS: Progression-free survival

\section{Acknowledgements}

Written consent to publish the study was obtained from the patients. We thank the "Struktur- and Innovationsfonds für die Forschung BadenWürttemberg" (SI-BW) and the DFG for financing NMR instrumentation, which is operated within the MagRes Center of the University of Freiburg.

\section{Authors' contributions}

WM drafted the manuscript and participated in the data collection. KD, JW, and NP conducted the laboratory tests. DD participated in the preparation of the manuscript and in the data collection. MTK participated in the design of the figures. OS, SW, and JB participated in the preparation of this article by revising it with regard to important intellectual content. DHH and JG coordinated the study and revised the article for important intellectual content. All authors read and approved the final manuscript.

\section{Funding}

This study was funded by the German Cancer Society (Seeding Grand TII) and Müller-Fahnenberg-Stiftung. The funding institutions did not play any role in the planning of the study and the collection, analysis and interpretation of the data, nor in the writing of the manuscript. Open Access funding enabled and organized by Projekt DEAL.

\section{Availability of data and materials}

Further information and requests for resources, raw data, and reagents should be directed to and will be fulfilled by DH Heiland (dieter.henrik. heiland@uniklinik-freiburg.de). A full table of all materials is given in the supplementary information.

\section{Ethics approval and consent to participate}

For this study, we included 43 patients who underwent surgery at the Department of Neurosurgery of the Medical Center, University of Freiburg. The local ethics committee of the University of Freiburg approved the data evaluation, imaging procedures, and experimental design (protocols 100020/ 09 and 5565/15). The methods were carried out in accordance with the approved guidelines. Written informed consent was obtained from each patient. The studies were approved by an institutional review board.

\section{Consent for publication}

All authors read and approved the final manuscript.

\section{Competing interests}

No potential conflicts of interest were disclosed by the authors.

\section{Author details}

${ }^{1}$ Department of Neurosurgery, University Medical Center Freiburg, Breisacher Straße 64, 79106 Freiburg, Germany. ${ }^{2}$ Faculty of Medicine, University of Freiburg, Freiburg im Breisgau, Germany. ${ }^{3}$ Institute of Physical Chemistry, Faculty of Chemistry and Pharmacy, University of Freiburg, Freiburg im Breisgau, Germany. ${ }^{4}$ Department of Neurosurgery, RWTH University, Aachen, Germany. ${ }^{5}$ Department of Neurosurgery, Cantonal Hospital St.Gallen, st. gallen, switzerland. 
Received: 29 September 2020 Accepted: 8 February 2021

Published online: 01 March 2021

\section{References}

1. Rogers L, et al. Meningiomas: knowledge base, treatment outcomes, and uncertainties. A RANO review. J Neurosurg. 2015;122:4-23.

2. Louis DN, et al. The 2016 World Health Organization classification of tumors of the central nervous system: a summary. Acta Neuropathol. 2016;131:803-20.

3. Clark VE, et al. Recurrent somatic mutations in POLR2A define a distinct subset of meningiomas. Nat Genet. 2016;48:1253-9.

4. Brastianos PK, et al. Genomic sequencing of meningiomas identifies oncogenic SMO and AKT1 mutations. Nat Genet. 2013;45:285-9.

5. Pfisterer WK, et al. Implicating chromosomal aberrations with meningioma growth and recurrence: results from FISH and MIB-I analysis of grades I and II meningioma tissue. J Neuro-Oncol. 2008:87:43-50.

6. Gao F, et al. DNA methylation in the malignant transformation of meningiomas. PLoS One. 2013;8:e54114.

7. Goutagny $\mathrm{S}$, et al. High incidence of activating TERT promoter mutations in meningiomas undergoing malignant progression. Brain Pathol. 2014;24:184-9.

8. Dunn J, et al. Integration and comparison of transcriptomic and proteomic data for meningioma. Cancers (Basel). 2020;12:3270.

9. Monleón $\mathrm{D}$, et al. Metabolic aggressiveness in benign meningiomas with chromosomal instabilities. Cancer Res. 2010;70:8426-34.

10. Bender $\mathrm{L}$, et al. Metabolomic profile of aggressive Meningiomas by using high-resolution magic angle spinning nuclear magnetic resonance. J Proteome Res. 2020:19:292-9.

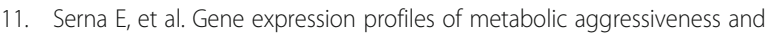
tumor recurrence in benign meningioma. PLoS One. 2013;8:e67291.

12. Bosnyák E, et al. Molecular imaging correlates of tryptophan metabolism via the kynurenine pathway in human meningiomas. Neuro-Oncology. 2015;17: 1284-92.

13. Zitron IM, Kamson DO, Kiousis S, Juhász C, Mittal S. In vivo metabolism of tryptophan in meningiomas is mediated by indoleamine 2,3-dioxygenase 1. Cancer Biol Ther. 2013;14:333-9.

14. Beckonert $\mathrm{O}$, et al. Metabolic profiling, metabolomic and metabonomic procedures for NMR spectroscopy of urine, plasma, serum and tissue extracts. Nat Protoc. 2007;2:2692-703.

15. Hwang T-L. Water Suppression that Works. Excitation sculping using Arbitrary Waveforms and plused fields gradients. J Magn Reson. 1995;112: 275-9.

16. Heiland $\mathrm{DH}$, et al. The integrative metabolomic-transcriptomic landscape of glioblastome multiforme. Oncotarget. 2017:8:49178-90.

17. Hao J, et al. Bayesian deconvolution and quantification of metabolites in complex 1D NMR spectra using BATMAN. Nat Protoc. 2014;9:1416-27.

18. Hastie TJ, Narasimhan B, Tibshirani RJ, Chu G. Predictive analysis of microarrays; 2002. p. 1-41

19. McCullagh, P., Nelder, J. A. Generalized Linear Models. 500 (Chapman \& Hall, 1989).

20. Langfelder P, Horvath S. WGCNA: an R package for weighted correlation network analysis. BMC Bioinformatics. 2008:9:559.

21. Holtman IR, et al. Induction of a common microglia gene expression signature by aging and neurodegenerative conditions: a co-expression meta-analysis. Acta Neuropathol Commun. 2015;3:31.

22. lancu OD, et al. Cosplicing network analysis of mammalian brain RNA-Seq data utilizing WGCNA and mantel correlations. Front Genet. 2015;6:174.

23. Heiland $\mathrm{DH}$, et al. Integrative network-based analysis of magnetic resonance spectroscopy and genome wide expression in Glioblastoma multiforme. Sci Rep. 2016;6:29052.

24. Luo W, Brouwer C. Pathview: an R/bioconductor package for pathwaybased data integration and visualization. Bioinformatics. 2013;29:1830-1.

25. Rogers $\mathrm{CL}$, et al. Pathology concordance levels for meningioma classification and grading in NRG oncology RTOG trial 0539. NeuroOncology. 2016;18:565-74.

26. Amelio I, Cutruzzolá F, Antonov A, Agostini M, Melino G. Serine and glycine metabolism in cancer. Trends Biochem Sci. 2014;39:191-8.

27. Gut $P$, Verdin $E$. The nexus of chromatin regulation and intermediary metabolism. Nature. 2013;502:489-98.

28. Cheng L, Chang I-W, Louis D, Gonzalez G. Correlation of high-resolution magic angle spinning proton magnetic resonance spectroscopy with histopathology of intact human brain tumor Specimens1. Cancer Res. 1998; 58:1825-32.
29. Pfisterer WK, et al. Using ex vivo proton magnetic resonance spectroscopy to reveal associations between biochemical and biological features of meningiomas. Neurosurg Focus. 2010;28:E12.

30. Pfisterer WK, et al. Fluorescent in situ hybridization and ex vivo $1 \mathrm{H}$ magnetic resonance spectroscopic examinations of meningioma tumor tissue: is it possible to identify a clinically-aggressive subset of benign meningiomas? Neurosurgery. 2007;61:1048-59; discussion 1060.

31. Gillies RJ, Barry JA, Ross BD. In vitro and in vivo $13 \mathrm{C}$ and 31P NMR analyses of phosphocholine metabolism in rat glioma cells. Magn Reson Med. 1994; 32:310-8.

32. Preul $\mathrm{M}$, et al. Accurate, nonivasive diagnosis of human brain tumors by using proton magnetic resonance spectroscopy. Nat Med. 1996;2:323-5.

33. Mishra G, Phatak S, Kakde D. Role of magnetic resonance spectroscopy in classifying MENINGIOMAS with HIST op a THOLOGICAL CORRELA TION. Int J Sci Res. 2018;7:2277-8179.

34. Mefford IN, Barchas JD. Determination of tryptophan and metabolites in rat brain and pineal tissue by reversed-phase high-performance liquid chromatography with electrochemical detection. J Chromatogr. 1980;180: 187-93.

35. Metz R, et al. Novel tryptophan catabolic enzyme IDO2 is the preferred biochemical target of the antitumor indoleamine 2,3-dioxygenase inhibitory compound D-1-methyl-tryptophan. Cancer Res. 2007:67:7082-7.

36. de Jong RA, et al. Serum tryptophan and kynurenine concentrations as parameters for indoleamine 2,3-dioxygenase activity in patients with endometrial, ovarian, and vulvar cancer. Int J Gynecol Cancer. 2011;21:1320-7.

37. Sucher $\mathrm{R}$, et al. IDO-mediated tryptophan degradation in the pathogenesis of malignant tumor disease. Int J Tryptophan Res. 2010;3:113-20.

38. Prendergast G, Cancer C. Why tumours eat tryptophan. Nature. 2011;478: 192-4.

\section{Publisher's Note}

Springer Nature remains neutral with regard to jurisdictional claims in published maps and institutional affiliations.
Ready to submit your research? Choose BMC and benefit from:

- fast, convenient online submission

- thorough peer review by experienced researchers in your field

- rapid publication on acceptance

- support for research data, including large and complex data types

- gold Open Access which fosters wider collaboration and increased citations

- maximum visibility for your research: over $100 \mathrm{M}$ website views per year

At $\mathrm{BMC}$, research is always in progress.

Learn more biomedcentral.com/submissions 